\title{
Modelos de regressão aleatória na avaliação da produção de leite em cabras da raça Saanen
}

\author{
Gilberto Romeiro de Oliveira Menezes ${ }^{1}$, Robledo de Almeida Torres ${ }^{2}$, José Lindenberg \\ Rocha Sarmento ${ }^{3}$, Marcelo Teixeira Rodrigues ${ }^{2}$, Luiz Fernando Brito ${ }^{4}$, Paulo Sávio Lopes ${ }^{2}$, \\ Felipe Gomes da Silva ${ }^{5}$
}

\footnotetext{
1 Pós-Graduação em Genética e Melhoramento, UFV. Bolsista do CNPq.

2 Departamento de Zootecnia, UFV. Bolsista do CNPq.

${ }^{3}$ Departamento de Zootecnia, UFPI

${ }^{4}$ Graduação em Zootecnia, UFV. Bolsista de Iniciação Científica do CNPq.

${ }^{5}$ Graduação em Agronomia, UFV. Bolsista de Iniciação Científica do CNPq.
}

RESUMO - Foram utilizados 10.238 registros semanais de produção de leite no dia do controle leiteiro provenientes de 388 primeiras lactações de cabras da raça Saanen visando comparar diferentes modelos de regressão aleatória (MRA). Primeiramente, foram comparados cinco modelos, cujos termos exponenciais da função de Wilmink assumiram os seguintes valores $-0,0350 ;-0,0500 ;-0,0565 ;-0,0680$ e $-0,1000$ (W0350, W0500, W0565, W0680 e W1000, respectivamente), considerando-se homogeneidade de variância residual ao longo da lactação. No modelo W0500, o valor $-0,0500$ foi mantido, enquanto nos modelos W0350, W0565, W0680 e W1000 foram usados os valores $-0,0350 ;-0,0565$; $-0,0680$; e $-0,1000$, respectivamente, em substituição ao valor -0,0500, proposto no modelo original utilizado para bovinos de leite. Escolhido o melhor modelo, segundo o ln L, foram avaliadas, pelos critérios AIC, BIC e ln L, a homogeneidade e heterogeneidade da variância residual: homogeneidade, duas, três, quatro, cinco e seis classes ao longo da lactação. De acordo com o critério usado, o modelo W0350 apresenta o melhor ajuste dentre os avaliados. Com relação à variância residual, a utilização de seis classes ao longo da lactação é indicada pelos critérios AIC, BIC, ln L e teste de razão de verossimilhança. As estimativas de herdabilidade ao longo da lactação, para o melhor modelo, variam de 0,07 (2aㅡ semana de lactação) a 0,25 (20aㅡ semana de lactação).

Palavras-chave: função de Wilmink, leite, modelagem, produção de leite no dia do controle, variância residual

\section{Random regression models in the milk yield evaluation in Saanen goats}

\begin{abstract}
It was used 10,238 weekly test day records from 388 first lactations of Saanen goats with the objective of comparing random regression models (RRM). Firstly, it was compared five models, whose exponential terms of Wilmink function assumed the following values: $-0.0350 ;-0.0500 ;-0.0565 ;-0.0680$ and -0.1000 (W0350, W0500, W0565, W0680 and W1000, respectively) by considering homogeneity of residual variance over the lactation period. The value -0.0500 was kept in the model W0500 whereas models W0350, W0565, W0680 and W1000 used values $-0.0350 ;-0.0565 ;-0.0680$ and -0.1000 , respectively, replacing the value -0.0500 , proposed by the original model used in dairy cattle. After choosing the best model according to ln L, homogeneity and heterogeneity for residual variance: homogeneity, two classes, three classes, four classes, five classes and six classes along the lactation were evaluated by using AIC, BIC and ln L criteria. According to criterion used, W0350 presents the best fit among the evaluated models. With regard to residual variance, the use of six classes over lactation is indicated by AIC, BIC, ln L and likelihood ratio test. Heritability estimates over lactation, for the best model, ranges from 0.07 ( $2^{\text {nd }}$ lactation week) to 0.25 ( $20^{\text {th }}$ lactation week).
\end{abstract}

Key Words: milk, milk yield on test day, modelling, residual variance, Wilmink function

\section{Introdução}

Nos últimos anos, os modelos de regressão aleatória (MRA) têm-se tornado o procedimento padrão para análises de dados longitudinais provenientes da produção animal (Meyer, 2005). Todavia, em caprinos leiteiros, poucos estudos têm sido desenvolvidos, o que reflete a necessidade de pesquisas buscando os devidos ajustes em função das particularidades intrínsecas à espécie, como intervalos de tempo entre as medidas, definição de subclasses para a curva fixa e da ordem de ajuste da regressão aleatória (Sarmento et al., 2008).

Em trabalhos avaliando a produção de leite no dia do controle em bovinos leiteiros utilizando MRA, a modelagem 
das curvas de regressão fixa e aleatórias genética aditiva e de ambiente permanente tem sido feita utilizando a função paramétrica de Wilmink (1987) e algumas modificações desta (Brotherstone et al., 2000; Jakobsen et al., 2002; Freitas, 2003). Breda et al. (2006), em trabalho com cabras da raça Alpina, avaliaram essa função e algumas modificações, relatando o melhor ajuste ao utilizar a função original. No entanto, estudos que orientem a modelagem de MRA na avaliação genética de cabras da raça Saanen não foram encontrados na literatura consultada.

A adequada modelagem da variância residual (variância de ambiente temporário) é outro aspecto fundamental para uma maior acurácia em avaliações genéticas sob MRA. De acordo com El Faro \& Albuquerque (2003), a modelagem, considerando as variâncias residuais heterogêneas entre classe, pode melhorar a partição da variação total, mas proporciona um aumento no número de parâmetros a serem estimados no processo de maximização da função de verossimilhança. Estudos têm proposto a utilização de um número reduzido de classes de variâncias residuais, por intermédio do agrupamento de classes que contenham variações semelhantes (El Faro \& Albuquerque, 2003; Freitas, 2003; Chaves, 2005; Assis et al., 2006; Breda et al., 2006).

Assim, o objetivo deste trabalho foi comparar a função paramétrica de Wilmink (1987) e algumas modificações desta na modelagem das curvas de regressão fixa e aleatórias genética aditiva e de ambiente permanente, além da homogeneidade e heterogeneidade de variância residual, a fim de se obter o modelo mais adequado a ser usado na descrição da variação genética da curva de lactação de cabras da raça Saanen, para posterior utilização em programas de avaliação genética.

\section{Material e Métodos}

Foram analisados 10.238 registros semanais de produção de leite no dia do controle (PLDC) provenientes de 388 primeiras lactações de cabras da raça Saanen, sendo que estas foram divididas em dois grupos genéticos: puro Saanen (243 cabras - 62,6\%) e mestiço Saanen (145 cabras - 37,4\%) (Tabela 1). Os animais utilizados pertencem ao rebanho caprino do Departamento de Zootecnia, da Universidade Federal de Viçosa.

Os controles foram registrados semanalmente, oriundos de ordenha mecânica, realizada duas vezes ao dia. Os animais foram mantidos em baias coletivas sob o sistema de estabulação livre. Os dados foram coletados entre os anos de 1999 e 2007. O banco de dados inicial era composto por 17.104 registros de PLDC, porém foram considerados os registros de controles da $2^{\underline{a}}$ até $39 \underline{a}$ semanas de lactação. Além disso, foram excluídos da análise animais com produções superiores ou inferiores a três desvios-padrão em relação à média de produção de leite na semana da lactação, com idade ao parto inferior a 10 meses ou superior a 30 meses, produções que ocorreram em grupos contemporâneos (ano-quizena do controle) com menos de três cabras e lactações com menos de seis controles (Tabela 1; Figura 1). A matriz dos numeradores dos coeficientes de parentesco continha 538 animais.

Tabela 1 - Número de controles leiteiros, médias e respectivos desvios-padrão para produção de leite na semana do controle da primeira lactação de cabras da raça Saanen

\begin{tabular}{|c|c|c|c|c|c|c|c|}
\hline Semana & NCL* & Média (kg) & $\mathrm{DP} * *$ & Semana & NCL* & Média (kg) & $\mathrm{DP} * *$ \\
\hline 2 & 197 & 1,88 & 0,65 & 21 & 303 & 1,80 & 0,80 \\
\hline 3 & 248 & 2,06 & 0,77 & 22 & 280 & 1,69 & 0,76 \\
\hline 4 & 283 & 2,15 & 0,80 & 23 & 269 & 1,73 & 0,72 \\
\hline 5 & 280 & 2,21 & 0,83 & 24 & 256 & 1,75 & 0,77 \\
\hline 6 & 335 & 2,22 & 0,82 & 25 & 302 & 1,61 & 0,71 \\
\hline 7 & 324 & 2,20 & 0,81 & 26 & 288 & 1,50 & 0,64 \\
\hline 8 & 280 & 2,20 & 0,83 & 27 & 292 & 1,58 & 0,72 \\
\hline 9 & 330 & 2,21 & 0,88 & 28 & 277 & 1,52 & 0,67 \\
\hline 10 & 343 & 2,17 & 0,86 & 29 & 253 & 1,44 & 0,69 \\
\hline 11 & 340 & 2,17 & 0,95 & 30 & 229 & 1,51 & 0,68 \\
\hline 12 & 325 & 2,08 & 0,85 & 31 & 240 & 1,47 & 0,66 \\
\hline 13 & 286 & 2,21 & 0,88 & 32 & 254 & 1,43 & 0,71 \\
\hline 14 & 308 & 2,12 & 0,87 & 33 & 243 & 1,41 & 0,72 \\
\hline 15 & 332 & 2,05 & 0,84 & 34 & 202 & 1,39 & 0,66 \\
\hline 16 & 286 & 2,12 & 0,81 & 35 & 190 & 1,48 & 0,72 \\
\hline 17 & 315 & 1,91 & 0,81 & 36 & 172 & 1,56 & 0,72 \\
\hline 18 & 301 & 1,88 & 0,83 & 37 & 170 & 1,40 & 0,70 \\
\hline 19 & 293 & 1,91 & 0,84 & 38 & 168 & 1,41 & 0,73 \\
\hline 20 & 298 & 1,91 & 0,75 & 39 & 146 & 1,49 & 0,71 \\
\hline
\end{tabular}

* NCL $=$ número de controles leiteiros.

**DP $=$ desvio-padrão. 


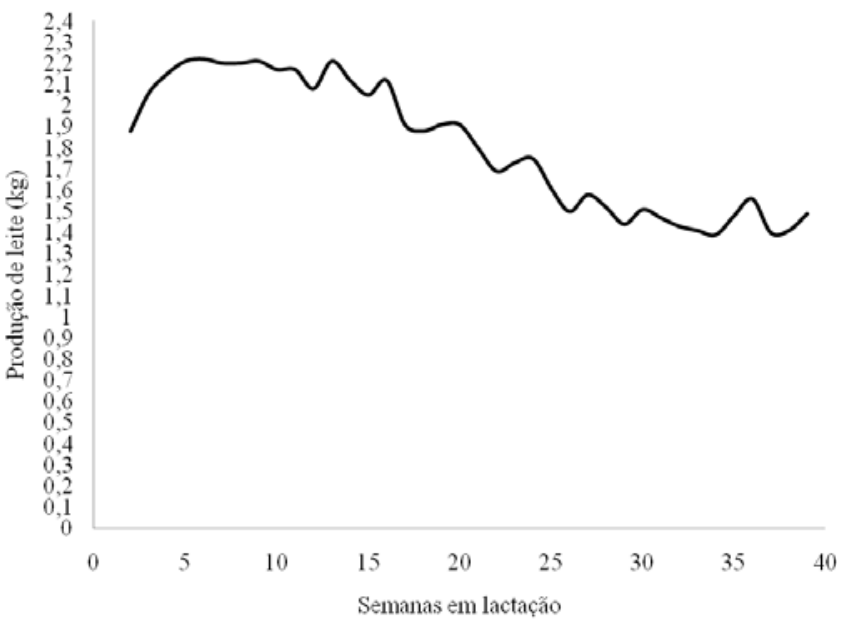

Figura 1 - Curva de lactação média traçada usando as produções de leite no dia do controle médias.

As análises foram realizadas por meio de um modelo animal unicaracterístico de regressão aleatória. A função de Wilmink (1987), que foi a base para comparações, e que é utilizada na descrição dos efeitos genético aditivo e de ambiente permanente do MRA, é representada por:

$$
\mathrm{y}=\mathrm{a}_{1}+\mathrm{a}_{2} \mathrm{t}+\mathrm{a}_{3} \mathrm{e}^{-0,05 \mathrm{t}}
$$

em que $\mathrm{y}=$ quantidade de leite produzida, em kg, no dia t; $\mathrm{a}_{1}=$ nível de produção; $\mathrm{a}_{2}=$ decréscimo da produção após o pico; $\mathrm{a}_{3}=$ aumento da produção até o pico; e o valor $-0,05$ = momento do pico, em torno de 50 dias após o parto (Wilmink, 1987).

Primeiramente, foi avaliada a modelagem das regressões fixa e aleatórias genéticas aditivas e de ambiente permanente sob homogeneidade de variância residual.

Cinco modelos de regressão aleatória foram comparados a partir de modificações no termo exponencial $\left(\mathrm{a}_{3} \mathrm{e}^{-0,05 t}\right)$ da função de Wilmink (1987). No modelo W0500, o valor $-0,0500$ foi mantido, enquanto nos modelos W0350, W0565, W0680 e W1000 foram usados os valores - 0,0350 (Schaeffer et al., 2000), -0,0565 (Breda et al., 2006), $-0,0680$ e-0,1000 (Brotherstone et al., 2000), respectivamente, em substituição ao valor -0,0500, que é o padrão da função de Wilmink (1987). Foi adotada a mesma notação para os cinco modelos:

$\mathrm{y}_{\mathrm{ij}}=\mathrm{F}+\sum_{m=1}^{3} b_{m} Z_{m}\left(t_{i j}\right)+\sum_{m=1}^{3} a_{i m} Z_{m}\left(t_{i j}\right)+\sum_{m=1}^{3} p_{i m} Z_{m}\left(t_{i j}\right)+e_{i j}$

em que $\mathrm{y}_{\mathrm{ij}}=$ produção de leite no controle j da cabra i; $\mathrm{F}$ = conjunto de efeitos fixos, constituído por grupo contemporâneo (ano-quinzena do controle-176 subclasses), grupo genético (puro e mestiço Saanen), tipo de parto (simples e duplo) e pela covariável idade da cabra ao parto, efeitos linear e quadrático; $b_{\mathrm{m}}=\mathrm{m}^{\text {ésimo }}$ coeficiente de regressão fixo da produção no dia do controle sobre a função utilizada para modelar a curva média de lactação da população; $a_{\text {im }}$ e $\mathrm{P}_{\text {im }}=$ coeficientes de regressão genético aditivo e de ambiente permanente, respectivamente, associados aos coeficientes específicos da função de Wilmink (1987) e modificações, que podem ser representados por $Z_{m}^{\prime}=\left(Z_{1} Z_{2} Z_{3}\right)^{\prime}=(1 \mathrm{td})^{\prime}$, sendo $d=e^{-w t}$ com $\mathrm{w}$ assumindo os valores $-0,0350,-0,0500,-0,0565,-0,0680 \mathrm{e}$ $-0,1000$ de acordo com a variação da função usada e, t e m, respectivamente, o período de dias em lactação após o parto e o mésimo parâmetro da função; $\mathrm{e}_{\mathrm{ij}}$ é o erro aleatório associado a cada controle $\mathrm{j}$ da cabra $\mathrm{i}$.

O modelo (2) pode ser descrito com a notação matricial: $\mathrm{y}=\mathrm{Xb}+\mathrm{Za}+\mathrm{Wp}+\mathrm{e}$,

em que: $\mathrm{y}=$ vetor das $\mathrm{N}$ observações, medidas em $\mathrm{N}_{\mathrm{d}}$ animais com registros; $b$ = vetor que inclui o conjunto de efeitos fixos (F) e os coeficientes de regressão fixa, específicos da função de Wilmink (1987) e modificações; a = vetor de soluções para os coeficientes de regressão aleatórios genéticos aditivos; $\mathrm{p}$ = vetor de soluções para os coeficientes de regressão aleatórios de ambiente permanente; $\mathrm{e}=$ vetor dos N diferentes resíduos; X, Z e W = matrizes de incidência dos efeitos fixos, genético aditivo e de ambiente permanente, respectivamente.

O número de linhas do vetor a é $\mathrm{k}_{\mathrm{a}} \times \mathrm{N}_{\mathrm{a}}$, sendo que $\mathrm{k}_{\mathrm{a}}$ representa o número de parâmetros da função de regressão para o efeito genético aditivo, $\mathrm{N}_{\mathrm{a}}$, o número de animais na matriz dos numeradores dos coeficientes de parentesco. $\mathrm{O}$ vetor $\mathrm{p}$ tem número de linhas igual à $\mathrm{k}_{\mathrm{p}} \mathrm{x} \mathrm{N}_{\mathrm{d}}$, sendo que $\mathrm{k}_{\mathrm{p}}$ representa o número de parâmetros da função de regressão para o efeito de ambiente permanente e $\mathrm{N}_{\mathrm{d}}$ o número de animais com registros.

Para os cinco modelos, foram admitidas as seguintes pressuposições:

$\left\{\begin{array}{l}\mathrm{a} \\ \mathrm{p} \\ \mathrm{e}\end{array}\right\} \sim \mathrm{N}(0, \mathrm{~V})$ em que $\mathrm{V}=\left\{\begin{array}{ccc}\mathrm{K}_{\mathrm{a}} \otimes \mathrm{A} & 0 & 0 \\ 0 & \mathrm{~K}_{\mathrm{p}} \otimes \mathrm{I} & 0 \\ 0 & 0 & \mathrm{R}\end{array}\right\}$

em que $K_{a}=$ matriz de covariância genética entre os coeficientes de regressão aleatória, considerados como sendo os mesmos para todas as cabras; $\mathrm{A}=$ matriz dos numeradores dos coeficientes de parentesco entre os animais, que neste trabalho foi composta por 538 animais; $\otimes$ = produto direto; $K_{p}=$ matriz de covariância de ambiente permanente entre os coeficientes de regressão aleatória; $\mathrm{I}$ = matriz identidade; $\mathrm{R}$ = matriz diagonal de variância residual, sob homogeneidade de variância residual ao longo da lactação.

Após a escolha da melhor função dentre as avaliadas sob homogeneidade de variância residual, foram avaliados seis modelos, os quais utilizavam a função escolhida 
anteriormente para descrever as curvas fixa e aleatórias genética aditiva e de ambiente permanente e, se diferenciaram pelo número de classes de variância residual assumidas ao longo da lactação, em que se assumiu homogeneidade de variância dentro de classe e heterogeneidade entre classes.

Os seis modelos avaliados foram:

CLA1 - homogeneidade de variância residual;

CLA2 - duas classes (2ㅁa a $20 \underline{a}$ e $21 \underline{a}$ a $39 \underline{a}$ semanas de lactação);

CLA3 - três classes ( $2^{\underline{a}}$ a $11^{\underline{a}}, 12^{\underline{a}}$ a $21^{\underline{a}}$ e $22^{\underline{a}}$ a $39^{\underline{a}}$ semanas de lactação);

CLA4 - quatro classes (2 $2^{\underline{a}}$ a 9a, $10^{\underline{a}}$ a $17 \underline{a}, 18^{\underline{a}}$ a $25^{\underline{a}}$ e $26^{\underline{a}}$ a 39a semanas de lactação);

CLA5 - cinco classes ( $2^{\underline{a}}$ a $8^{\underline{a}}, 9^{\underline{a}}$ a $15^{\underline{a}}, 16^{\underline{a}}$ a $23^{\underline{a}}, 24^{a}$ a $31^{\mathrm{a}}$ e $32^{\underline{a}}$ a $39^{\mathrm{a}}$ semanas de lactação);

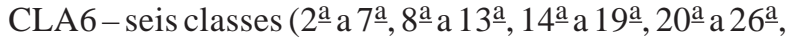

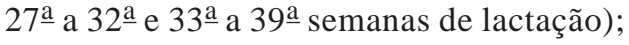

em que CLA = classe e 1, 2, 3, 4, 5 e 6 = número de classes de variância residual.

O critério utilizado para a formação das classes de variância residual foi dividir a lactação de forma que as classes englobassem semelhante número de semanas.

A matriz R citada anteriormente, em condições de heterogeneidade de variância residual, foi redefinida como $\mathrm{R}=\operatorname{diag}\left\{\mathrm{I}_{\mathrm{e} \mathrm{i}}^{2}\right\}$, em que $\sigma_{\mathrm{e} i}^{2}=$ variância da classe de resíduo $\mathrm{i}$.

As (co) variâncias entre os coeficientes de regressão aleatória genéticos aditivos e de ambiente permanente foram estimadas pelo método da máxima verossimilhança restrita livre de derivadas (REML), usando o programa DXMRR do DFREML (Meyer, 1998). A maximização da verossimilhança foi realizada usando o algoritmo POWELL (Powell, 1964). O critério de convergência utilizado foi $10^{-9}$.

As comparações entre os modelos foram feitas pelo Critério de Informação de Akaike (AIC), Critério de Informação Bayesiano de Schwarz (BIC), Logaritmo neperiano da função de verossimilhança (ln L) e teste da razão de verossimilhança (TRV), a 1\% de probabilidade (apenas para modelos aninhados). Os critérios AIC e BIC foram usados apenas na comparação de modelos com número diferentes de parâmetros. Para AIC o valor é calculado como se segue:

AIC $=-2 \ln \mathrm{L}+2 \mathrm{p}$

e para BIC, é feito através de:

$\mathrm{BIC}=-2 \ln \mathrm{L}+\mathrm{p} \ln (\mathrm{N}-\mathrm{r})$,

em que: $\mathrm{p}$ = número de parâmetros do modelo; $\mathrm{N}$ = número total de observações e r = posto da matriz X, que é a matriz de incidência para os efeitos fixos. Menores valores para AIC e BIC e, maiores de ln L indicam melhor ajuste do modelo.

\section{Resultados e Discussão}

A partir dos resultados, os autores verificaram que o modelo W0350, o qual utiliza o valor $-0,0350$ em substituição ao -0,0500 no termo exponencial $\left(\mathrm{a}_{3} \mathrm{e}^{-0,05 t}\right)$ da função de Wilmink (1987), apresentou o melhor ajuste no valor de ln L (Tabela 2). Uma possível explicação para o melhor ajuste não ter sido proporcionado pelo modelo W0500 (modelo original) é que o valor -0,0500 é referente a um pico de lactação próximo aos 50 dias após o parto (Wilmink, 1987). No presente estudo, o pico de lactação ocorreu em torno dos 40 dias de lactação (Tabela 1), sugerindo que o valor -0,0350 estaria mais proximamente relacionado com o pico de lactação dos animais do rebanho em estudo (40 dias), entretanto, é importante ressaltar que não se deve assumir, a princípio, que o melhor valor a ser usado no termo exponencial da função de Wilmink (1987) seja aquele cujo valor após a vírgula seja igual ao pico de lactação, i.e. assumir, por exemplo, que o valor $-0,0600$ seja o mais adequado quando se tiver pico de lactação aos 60 dias.

Na literatura consultada, não foram encontrados trabalhos utilizando esta metodologia, com cabras da raça Saanen que permitissem a comparação dos resultados encontrados. No entanto, para a raça Alpina, Breda et al. (2006) ao avaliarem os modelos W0500, W0565 e W1000 no ajuste das curvas fixa e aleatórias genética aditiva e de ambiente permanente, sob homogeneidade de variância residual, indicaram o modelo W0500 como o mais adequado. Este resultado corrobora os encontrados no presente estudo, uma vez que, ao desconsiderar o modelo W03500, o W0500 passaria a ser o melhor, com base nos critérios utilizados (Tabela 2). A concordância entre os dois trabalhos pode ser explicada pela semelhança, em nível produtivo, entre as duas raças e pelo fato de os dois rebanhos estudados pertencerem a um mesmo criatório, gerando homogeneidade de manejo.

Tabela 2 - Logaritmo neperiano da função de máxima verossimilhança para os diferentes modelos

\begin{tabular}{ll}
\hline Modelo** & $\ln$ L*** \\
\hline W0350* & 3209,92 \\
W0500 & 3188,56 \\
W0565 & 3175,79 \\
W0680 & 3152,16 \\
W1000 & 3091,75 \\
\hline
\end{tabular}

* W0350, W0500, W0565, W0680 e W1000 representam, respectivamente, os modelos cujos os termos exponenciais da função de Wilmink assumiram os valores $-0,0350$; -0,0500; -0,0565; -0,068; e -0,1000.

** todos os cinco modelos tiveram 13 parâmetros estimados.

*** $\ln \mathrm{L}=$ logaritmo neperiano da função de máxima verossimilhança. 
Tabela 3 - Número de parâmetros estimados e critérios usados na comparação de seis diferentes modelos comparados quanto à modelagem da variância residual

\begin{tabular}{|c|c|c|c|c|c|c|}
\hline Modelo & $\mathrm{NP} * * *$ & $\ln \mathrm{L}^{* * *}$ & AIC $* * *$ & $\mathrm{BIC} * * *$ & $\mathrm{TRV} * * *$ & \\
\hline CLA $1 * *$ & 13 & 3209,93 & $-6393,85$ & $-6299,81$ & - & - \\
\hline CLA2 & 14 & 3267,20 & $-6506,41$ & $-6405,14$ & 114,56 & * $(2-1)$ \\
\hline CLA3 & 15 & 3273,85 & $-6517,70$ & $-6409,20$ & 13,29 & $*(3-2)$ \\
\hline CLA4 & 16 & 3278,57 & $-6525,14$ & $-6409,39$ & 9,43 & $*(4-3)$ \\
\hline CLA5 & 17 & 3279,94 & $-6525,89$ & $-6402,91$ & 2,75 & ns $\quad(5-4)$ \\
\hline CLA6 & 18 & 3295,28 & $-6554,56$ & $-6424,35$ & 30,67 & $*(6-5)$ \\
\hline
\end{tabular}

* significativo a $1 \%$ de probabilidade.; ns não-significativo.

** CLA1, CLA2, CLA3, CLA4, CLA5 e CLA6 representam os modelos em que se assumiu uma, duas, três, quatro, cinco e seis classes de variância residual, respectivamente. *** NP = número de parâmetros, ln L = logaritmo neperiano da função de máxima verossimilhança, AIC = critério de informação de Akaike, BIC = critério de informação bayesiano de Schwarz e TRV = teste da razão de verossimilhança.

De posse do modelo mais adequado (W0350) sob homogeneidade de variância residual, avaliou-se a modelagem da variância residual, considerando a variância como homogênea (CLA1) e heterogênea em classes (CLA2, CLA3, CLA4, CLA5 e CLA6) ao longo da lactação.

O modelo sob homogeneidade de variância residual (CLA1) apresentou o pior ajuste, como sugerido pelos valores dos critérios utilizados (Tabela 3). Este resultado é semelhante ao encontrado por Breda et al. (2006) que, ao avaliarem três estruturas para a variância residual (homogeneidade, três e quatro classes de resíduo ao longo da lactação), apontaram o modelo que desconsiderava a heterogeneidade de variância residual como o de pior ajuste. Outros autores (Rekaya et al., 2000; El Faro \& Albuquerque, 2003; Assis et al., 2006; Sarmento et al., 2008) também relataram variâncias residuais heterogêneas no decorrer da lactação, sendo que os dois primeiros trabalhos foram com bovinos e os dois últimos com caprinos. Essa heterogeneidade pode estar associada a diversos fatores, como período de gestação, condições do parto e duração do período seco, pois estes afetam a produção de leite ao longo da lactação, podendo gerar efeito de escala de medida. A introdução desses efeitos nas análises muitas vezes não é viável, em decorrência da ausência de informação, sendo necessário, portanto, considerar a existência de heterogeneidade residual ao longo da curva de lactação (Assis et al., 2006).

Por meio dos resultados do presente estudo observase que à medida que se aumentou o número de classes do resíduo, houve melhoria do ajuste, como indicado por todos os critérios, com exceção do TRV para os modelos CLA4 e CLA5, onde não houve diferença significativa ( $P>0,01$ ) entre os valores de ln L de CLA4 e CLA5 e, portanto, inexistência de diferença de qualidade de ajuste entre os dois (Tabela 3). Estes resultados são semelhantes aos apresentados por El Faro \& Albuquerque (2003) em bovinos da raça Caracu e Breda et al., (2006) em caprinos da raça Alpina, que também relataram essa tendência de melhoria de ajuste com o aumento do número de classes de resíduo. Uma explicação para essa tendência é que, com aumento do número de classes e conseqüente melhor partição da variação associada à curva da lactação, temse um maior refinamento na modelagem dos efeitos de ambiente temporário, acarretando partição mais precisa e acurada da variação total existente. Todavia, o aumento do número de classes deve ser feito com cautela, pois conduz a aumento do número de parâmetros a ser estimados, o que pode ocasionar dificuldades de convergência, especialmente em banco de dados com grande volume de observações.

Com base na análise dos resultados (Tabelas 2 e 3), o modelo CLA6, cuja modelagem das curvas fixa e aleatórias foi feita usando a função de Wilmink modificada (-0,0350 em vez de -0,0500 no termo exponencial) e assumiu-se heterogeneidade de variância residual com seis classes de resíduo ao longo da lactação, foi aquele que apresentou o melhor ajuste e, portanto, deve ser preferido nos estudos genéticos da curva de lactação de cabras da raça Saanen, utilizando MRA.

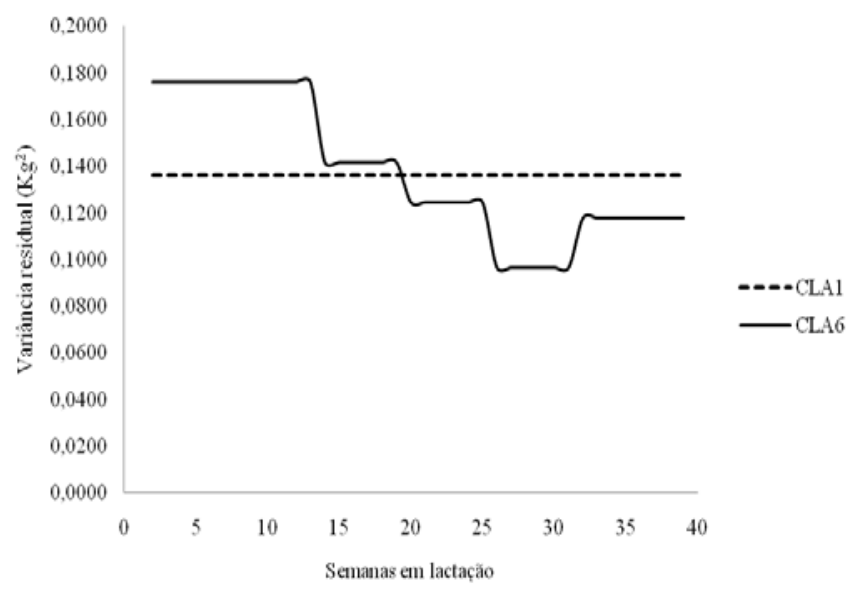

Figura 2 - Estimativas da variância residual para os modelos sob homogeneidade (CLA1) e heterogeneidade de variância residual com seis classes de resíduo (CLA6). 
Assumir homogeneidade de variância residual ao longo da lactação (CLA1) levou a subestimação da variância residual no início da lactação e a superestimação no final da lactação (Figura 2) em comparação à condição de heterogeneidade de variância (CLA6), a qual se mostrou ser a mais adequada neste estudo, segundo os critérios usados neste estudo. Estes resultados corroboram os encontrados por Sarmento et al. (2008), em estudo com cabras da raça Alpina.

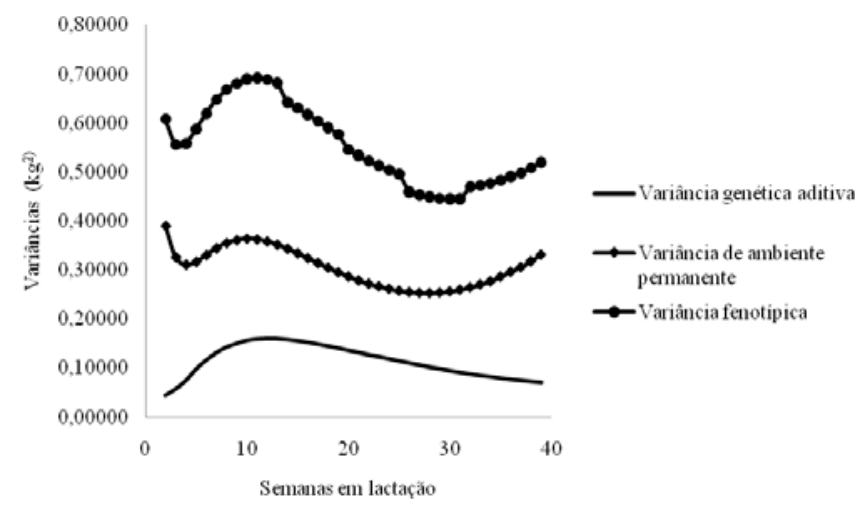

Figura 3 - Estimativas das variâncias genética aditiva, ambiente permanente e fenotípica da produção de leite no dia do controle para o modelo de melhor ajuste (CLA6).

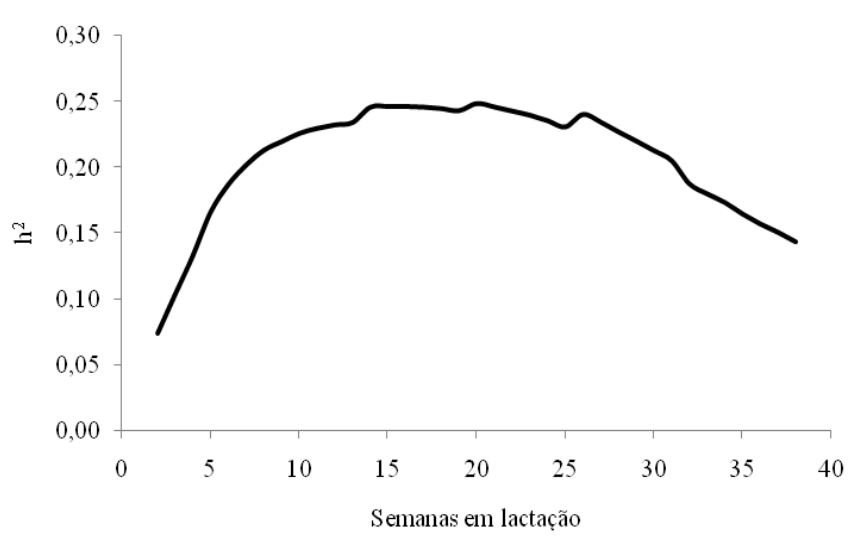

Figura 4 - Estimativas de herdabilidade $\left(h^{2}\right)$ para produção de leite no dia do controle para o modelo de melhor ajuste (CLA6).
Sarmento et al. (2008), utilizando diferentes MRA na modelagem da curva de lactação de cabras da raça Alpina, obtiveram curvas de variâncias semelhantes às do presente estudo (Figura 3), todavia, com estimativas de variâncias superiores.

A menor estimativa de herdabilidade $(0,07)$ foi encontrada na $2^{\underline{a}}$ semana de lactação, enquanto a maior $(0,25)$ foi encontrada na $20^{\underline{a}}$ semana (Figura 4). Estes valores foram inferiores aos encontrados por Assis et al. (2006) e Breda et al. (2006), que usando MRA, obtiveram estimativas de herdabilidade mínimas e máximas iguais a 0,09 (39a semana) e 0,32 ( $3^{\underline{a}}$ semana) e 0,12 (39ㅁa semana) e 0,51 ( $2^{\text {a }}$ semana), respectivamente, para cabras da raça Alpina. De maneira geral, foram observadas menores estimativas de herdabilidade no início e final da lactação e maiores para o meio desta (Figura 4), sugerindo que maiores ganhos genéticos seriam alcançados ao se utilizar a produção de leite em controles provenientes do meio da lactação como critério de seleção. Maior número de efeitos de meio agindo sobre o animal lactante nos limites da lactação (início e final), que aumentam a variância de ambiente temporário e, conseqüentemente, a variância fenotípica, pode ser a causa deste comportamento das estimativas de herdabilidade (Madsen, 1975, citado por Cobuci et al., 2004).

Todavia, diversas estimativas de herdabilidade ao longo da lactação são encontrados na literatura. Rekaya et al. (2000) relataram resultados semellhantes aos do presente estudo com bovinos da raça Holandesa, encontrando menores estimativas de herdabilidade no início e final da lactação e maiores para o meio desta. Por outro lado, El Faro \& Albuquerque (2003), com bovinos da raça Caracu, obtiveram resultados antagônicos aos deste trabalho, ou seja, maiores estimativas no início e final da lactação e menores no meio desta. Araújo et al. (2006) observaram estimativas de herdabilidade crescentes ao longo da lactação, em bovinos da raça Holandesa. Para caprinos da raça Alpina, Assis et al. (2006), Breda et al. (2006) e Sarmento et al. (2008) relataram estimativas da herdabilidade decrescentes ao

Tabela 4 - Estimativas de correlação genética (acima da diagonal) e fenotípica (abaixo da diagonal), entre as semanas em lactação, obtidas pelo modelo CLA6

\begin{tabular}{|c|c|c|c|c|c|c|c|c|c|}
\hline \multirow{2}{*}{$\begin{array}{l}\text { Semanas em } \\
\text { lactação }\end{array}$} & \multicolumn{9}{|c|}{ Semanas em lactação } \\
\hline & 2 & 5 & 10 & 15 & 20 & 25 & 30 & 35 & 39 \\
\hline 2 & - & 0,768 & 0,619 & 0,582 & 0,566 & 0,552 & 0,531 & 0,497 & 0,459 \\
\hline 5 & 0,552 & - & 0,978 & 0,964 & 0,951 & 0,932 & 0,897 & 0,840 & 0,776 \\
\hline 10 & 0,387 & 0,686 & - & 0,997 & 0,989 & 0,974 & 0,942 & 0,888 & 0,826 \\
\hline 15 & 0,346 & 0,668 & 0,757 & - & 0,997 & 0,986 & 0,961 & 0,914 & 0,858 \\
\hline 20 & 0,330 & 0,637 & 0,731 & 0,764 & - & 0,996 & 0,979 & 0,942 & 0,894 \\
\hline 25 & 0,311 & 0,578 & 0,669 & 0,716 & 0,747 & - & 0,993 & 0,968 & 0,931 \\
\hline 30 & 0,290 & 0,506 & 0,591 & 0,654 & 0,712 & 0,748 & - & 0,991 & 0,967 \\
\hline 35 & 0,244 & 0,387 & 0,458 & 0,532 & 0,611 & 0,676 & 0,752 & - & 0,999 \\
\hline 39 & 0,201 & 0,300 & 0,361 & 0,441 & 0,535 & 0,622 & 0,722 & 0,755 & - \\
\hline
\end{tabular}


longo da lactação. Diferentes estimativas de herdabilidade ao longo da lactação indicam diferenças nos ganhos genéticos ao se utilizar, como critério de seleção, produções de leite mensuradas em diferentes estádios da lactação. Além disso, diante da diversidade de formas de variação das estimativas de herdabilidade ao longo da lactação evidenciada por este estudo e aqueles encontrados na literatura, percebe-se a importância de se avaliar os parâmetros genéticos de uma população para o sucesso de um programa de melhoramento genético animal.

As correlações genéticas e fenotípicas estimadas entre as produções nos controles quando ajustado o modelo CLA6 são apresentadas na Tabela 4. As correlações genéticas variaram de 0,459 (estimativa obtida entre a 2 a e a 39a semana) a 0,999 (estimativa obtida entre a 35ㅁe e a 39aㅗ semana), demonstrando que, entre controles próximos, os valores foram os maiores, decrescendo continuamente conforme o aumento da distância entre os controles. Estes resultados corroboram os encontrados por Assis et al. (2006) em cabras da raça Alpina. Uma possível explicação para isso seria que a expressão dos genes ligados à produção de leite varia ao longo da lactação, sendo esta expressão mais semelhante para controles leiteiros próximos. Outra possibilidade seria que à medida que se aumenta o intervalo de controles, o grupo de genes ligados à produção leiteira torna-se mais diferente.

\section{Conclusões}

Modificações no termo exponencial da função de Wilmink podem promover melhor modelagem das curvas fixa e aleatórias genética aditiva e de ambiente permanente em avaliações da produção de leite em cabras da raça Saanen, usando modelos de regressão aleatória. Assumir heterogeneidade de variância residual é necessário para melhor partição das variâncias associadas à curva de lactação. Maiores ganhos genéticos podem ser alcançados ao se utilizar a produção de leite em controles provenientes do meio da lactação como critério de seleção, devido às suas maiores estimativas de herdabilidade.

\section{Agradecimentos}

Ao Dr. Roberto Augusto de Almeida Torres Júnior, pesquisador da Embrapa Gado de Corte, pelas importantes sugestões que contribuíram para a melhoria deste trabalho.

\section{Referências}

ARAÚJO, C.V.; TORRES, R.A.; COSTA, C.N. et al. Uso de modelos de regressão aleatória para descrever a variação genética da produção de leite na raça Holandesa. Revista Brasileira de Zootecnia, v.35, n.3, p.975-981, 2006.

ASSIS, G.M.L.; ALBUQUERQUE, L.G.; SARMENTO, J.L.R. et al. Estimação de parâmetros genéticos em caprinos leiteiros por meio da análise de regressão aleatória, utilizando a amostragem de Gibbs. Revista Brasileira de Zootecnia, v.35, n.3, p.706-714, 2006.

BREDA, F.C.; ALBUQUERQUE, L.G.; YAMAKI, M. et al. Estimação de parâmetros genéticos para produção de leite de cabras da raça Alpina. Revista Brasileira de Zootecnia, v.35, n.2, p.396-404, 2006.

BROTHERSTONE, S.; WHITE, I.M.S.; MEYER, K. Genetic modeling of daily yield using orthogonal polynomials and parametric curves. Animal Science, v.70, p.407-415, 2000.

CHAVES, L.C.S. Avaliação da persistência da lactação em vacas Girolando utilizando modelos de regressão aleatória. 2005. 86f. Dissertação (Mestrado em Zootecnia) - Universidade Federal de Viçosa, Viçosa, MG.

COBUCI, J.A.; EUCLYDES, R.F.; COSTA, C.N. et al. Análises da persistência na lactação de vacas da raça Holandesa, usando produção no dia do controle e modelo de regressão aleatória. Revista Brasileira de Zootecnia, v.33, n.3, p.546-554, 2004.

EL FARO, L.; ALBUQUERQUE, L.G. Utilização de modelos de regressão aleatória para produção de leite no dia do controle, com diferentes estruturas de variâncias residuais. Revista Brasileira de Zootecnia, v.32, n.5, p.1104-1113, 2003.

FREITAS, M.S. Utilização de modelos de regressão aleatória na avaliação genética de animais da raça Girolando. 2003. 78f. Dissertação (Mestrado em Zootecnia) - Universidade Federal de Viçosa, Viçosa, MG.

JAKOBSEN, J.H.; MADSEN, P.; JENSEN, J. et al. Genetic parameters for milk production and persistency for Danish Holstein estimated in random regression models using REML. Journal of Dairy Science, v.85, n.6, p.1607-1616, 2002.

MADSEN, O. A comparison of some suggested measures of persistency of milk yield in dairy cows. Animal Production v.20, p.191-197, 1975.

MEYER, K. DXMRR - A program to estimate covariance functions for longitudinal data by REML. In: WORLD CONGRESS OF GENETICS APPLIED TO LIVESTOCK PRODUCTION, 6. 1998, Armidale. Proceedings... Armidale: University of New England, 1998. (CD-ROM).

MEYER, K. Advances in methodology for random regression analyses. Australian Journal of Experimental Agriculture, v.45, p.847-858, 2005.

POWELL, M.J.D. An efficient method for finding the minimum of a function of several variables without calculating derivates. Computer Journal, v.7, p.155, 1964.

REKAYA, R.; CARABAÑO, M.J.; TORO, M.A. Assessment of heterogeneity of residual variance using changing points techniques. Genetics Selection Evolution, v.32, p.339-346, 2000.

SARMENTO, J.L.R.; ALBUQUERQUE, L.G.; TORRES, R.A. et al. Comparação de modelos de regressão aleatória para estimação de parâmetros genéticos em caprinos leiteiros. Revista Brasileira de Zootecnia, v.37, n.10, p.1788-1796, 2008.

SCHAEFFER, L.R.; JAMROZIK, J.; KISTEMAKER, G.J. et al. Experience with a test-day model. Journal of Dairy Science, v.83, p.1135-1144, 2000.

WILMINK, J.B.M. Adjustment of test-day milk, fat and protein yields for age, season and stage of lactation. Livestock Production Science, v.16, p.335-348, 1987. 